\author{
DANIEL SUTHERS, NATHAN DWYER, RICHARD MEDINA, RAVI \\ VATRAPU
}

\title{
EXPOSING INTERACTIONAL PROCESSES IN ONLINE LEARNING
}

\section{PREVIEW}

The interactional construction of meaning in collaborative learning is as important in online settings as it is face-to-face. Yet, due to the asynchronous and distributed nature of the online environment, online interaction is not as directly available as resource for intervention, analysis and reflection to teachers as it is in the classroom. A first step towards making online interaction visible is to capture it in a consolidated abstract transcript - a representation that can support visualization and analysis for researchers and practitioners. This chapter reports on a framework for analysis that we have developed that builds on such an artefact. The framework offers a representation, the contingency graph, that visualizes the interactional construction of meaning in distributed media and supports analysis of it. Examples are provided using data derived from asynchronous interaction of small groups in a collaborative knowledge-building environment.

\section{INTRODUCTION}

Today's schooling is not limited to the physical classroom, and has shifted to include online interactions as a significant component in both formal and informal education (Allen \& Seaman, 2005). Online learning is being implemented under a variety of "blended" learning models (Orey, McClendon, \& Branch, 2006) and K12 settings (Parker, 2000) as well as strictly online models in university education (Mayadas, 1997). Collaborative learning in online settings leverages both social processes of learning and representational resources for this learning (Dillenbourg, 2005; Suthers, in press). Yet, although collaborative inquiry learning is potentially equally valuable online as it is face-to-face, further work is needed to improve the representational resources that support negotiation of meaning online, and to develop effective instructional scenarios that leverage these resources in support of collaborative knowledge-construction processes and knowledge-building communities. A survey of the research on online learning (a compilation may be found at www.alnresearch.org, see also Moore, 2004) reveals that much of this

Pre-publication draft of Suthers, D. D., Dwyer, N., Medina, R., \& Vatrapu, R. (2009). Exposing interactional processes in online learning. In K. Kumpulainen, C. E. Hmelo-Silver \& M. César (Eds.), Investigating Classroom Interaction: Methodologies in Action (pp. 121-144). Rotterdam: Sense Publishers. 
research is concerned with establishing and securing an institutional role for online learning. Research directed towards that pragmatic and instrumental end attempts to demonstrate the equivalency of traditional face-to-face classroom learning and blended or online learning, based largely on measures of learning outcomes and students and faculty perceptions of satisfaction (Ramage, 2002). These are important measures for those concerned with the implementation of online learning as a customer oriented business, but less satisfying from a learning sciences standpoint. Exceptions in that literature include Aviv, Erlich, Ravid, \& Geva (2003) and Campos (2004). Like these authors, we seek to understand how learning is accomplished within and influenced by the affordances of online environments, and to apply this understanding to the design of improved online learning environments and activities for collaborative inquiry (Suthers, 2006b). Such an agenda must consider process data in addition to outcomes and satisfaction. Practitioners also need to observe the individual and collective activities of students and how these change in response to interventions such as adjustments to instructional resources and strategies. Therefore, tools that serve our research agenda can also ultimately assist educators in improving their practice through implicit or explicit forms of action research. But how can interaction that is distributed across time and media be made visible?

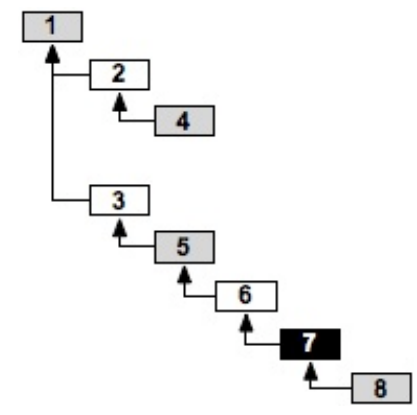

Figure 1. Reply structure of a threaded discussion

Consider two practical examples. In the first example, students have read some articles and are discussing them in threaded discussions. There are several discussion forums active, each discussion has multiple topic threads, and each thread splits into multiple subthreads (e.g., as shown in Figure 1). Participating students return over several days and may be active in different threads in each of their login sessions. How do we identify knowledge-construction processes? Who is building on whose ideas? What role is each participant playing? An example to be given later in this chapter will show that it is not sufficient to analyse merely the literal record that is left behind in one discussion. For a fuller understanding, one must consider other activity in addition to message posting, and consider activity across multiple forums (or more generally, multiple "locations" in the medium). 


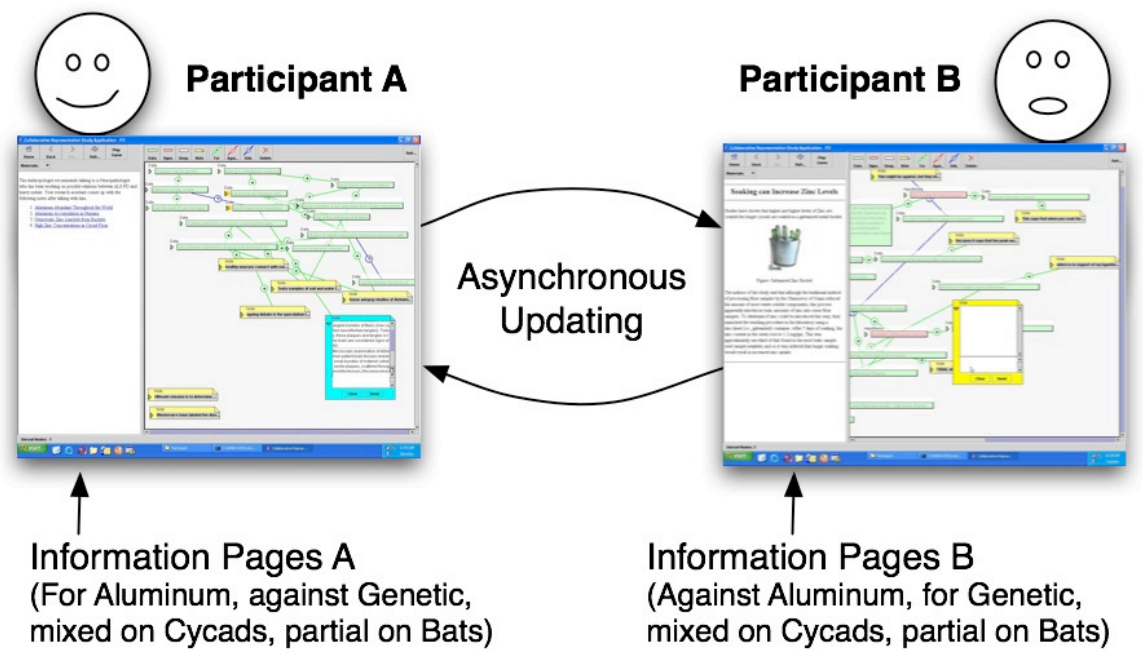

Figure 2. Interacting through graphical workspaces

Both researcher and practitioner need assistance in seeing the participatory structures that led to any given record of messages and replies.

In a line of research that originated in the Belvedere project (Suthers et al., 2001), we have been studying how knowledge-mapping environments can be designed to foster critical thinking and collaborative inquiry (Suthers \& Hundhausen, 2003; Suthers, Vatrapu, Medina, Joseph, \& Dwyer, 2008; Toth, Suthers, \& Lesgold, 2002). In the second example, students are interacting in a knowledge-mapping environment in which they access online information about a poorly understood but important disease, and consider and evaluate the evidence bearing on hypotheses about the cause of this disease in a graphical evidencemapping tool as well as a threaded discussion forum (Figure 2). As before, they are interacting asynchronously, but additionally their interaction is distributed across different types of media (the threaded discussion and the evidence mapping tool) as well as across discussion threads. Furthermore, the evidence-mapping environment provides a range of non-linguistic as well as linguistic expressive resources. Students can choose different shapes to represent the status of their statements, link data to hypotheses, and group or otherwise arrange these shapes in space for whatever purposes they see fit. Analytic methods that assume that interaction is language-based and consists of contribution-reply pairs will not capture all or even the majority of the relevant aspects of this kind of interaction. Significant actions and even interaction can take place through non-linguistic means (Suthers, 2006a).

In both of these examples, the concept of interaction is relevant, and it is important to uncover the actual procedures by which participants accomplish learning through the affordances of the media (Koschmann et al., 2005), but interaction takes different forms than those normally handled by the analytic 
traditions on which we draw. Methods for studying the interactional construction of meaning (e.g., Goodwin \& Heritage, 1990; Jordan \& Henderson, 1995) have largely been developed for brief episodes of face-to-face data, and require adaptation to online environments where media resources, time scale, and synchronicity differ (see Stahl, 2006b for an example of adaptation to synchronous chat and whiteboards). Analyses that are too closely tied to media representations may fail to identify interactional sources of coherence. As a simple example, consider again the reply structure of the threaded discussion shown in Figure 1. There appears to be two divergent lines of discussion, but an analysis to be presented in this paper shows that this is not the case. Additionally, most research on online learning is currently conducted in text-based tools. We need methods to study how richer representations might mediate online learning, as in the second example.

The immediate objective of the work reported in this paper is to make distributed and asynchronous interactions available for analysis. The long-term objective is to obtain a deep understanding of how learning is accomplished in technology-mediated settings by analysing computer-mediated interactions that span long durations of time and take place in different media among groups of various sizes. As a first step, we have developed an abstract transcript notation, the contingency graph, that provides a media-independent foundation for analysing how participants build on each others' contributions. Contingency graphs are grounded in identification of media coordinations and are intended to support identification of uptake as a bridge towards further analysis. The remainder of this paper briefly motivates and introduces the concept of uptake; describes the contingency graph as the basis for analysis of uptake (and ultimately meaningmaking) in online settings; and provides examples of this analysis applied to two sources of data (an online course discussion and a laboratory study of asynchronously interacting dyads, being the two examples just introduced).

\section{MOTIVATIONS}

Below we summarize the view of learning underlying our current work, and assess prevalent analysis methods and requirements for transcript representations in relation to our needs.

\section{Learning as an Interactive Process}

Although we believe that the framework we offer in this paper can support analyses under a variety of learning theories, the framework is motivated by our own views of learning, particularly as it takes place in social settings. Learning is conceived of not merely the transfer of information but rather as an interactional process of change. This conception of learning as interactional is compatible with theories of learning that identify individuals (Beck, 1997; Chi, Bassok, Lewis, Reimann, \& Glaser, 1989), socially embedded individuals (Doise \& Mugny, 1984; Vygotsky, 1978), social systems (Engestrom, 2001), or communities (Scardamalia 
\& Bereiter, 1991; Wenger, 1998) as the locus of change. Learning need not be the primary objective of an activity: it is a result of participants' attempts to make sense of a situation (Dervin, 2003). Meaning-making, as we call sense-making in this chapter, takes place at multiple levels: solving a problem, maintaining interpersonal relationships, and/or affirming identity in a community (Bronckart, 1995). To study learning in social settings we must necessarily study individual trajectories of meaning-making and how they intertwine in social practices of intersubjective meaning-making (Stahl, 2006a; Suthers, 2006b). Meaning is interactionally constructed and situated: the meaning of a given contribution (which may express an attitude and attentional orientation as well as information) is best understood as a function of its relationships to prior contributions and with respect to the physical and social context (Koschmann, Zemel, \& Stahl, 2004). Meaning-making is mediated by the physical and social environment in diverse ways (Hutchins, 1995; Wenger, 1998; Wertsch, 1998). As designers of media for online learning environments, this mediation gives us an avenue for influencing meaning-making and possibly learning through the socio-technical affordances of the tools that we design (P. Resnick, 2002; Suthers, 2006b).

\section{Analytic Approaches}

Motivated by this view of learning, it is natural to take an analytic approach that finds the significance of each act in the context of the unfolding interaction, such as Conversation Analysis (Goodwin \& Heritage, 1990; Sacks, Schegloff, \& Jefferson, 1974) and Interaction Analysis (Jordan \& Henderson, 1995), Typically, these methods repeatedly examine the micro-structure of short interaction segments to uncover the methods by which participants accomplish accountability in activity (Garfinkel, 1967). For examples applied to the analysis of learning, see (Baker, 2003; Koschmann \& LeBaron, 2003; Koschmann et al., 2005; Roschelle, 1996). This approach is a complement to the quantitative/statistical paradigm in which contributions (or elements of contributions) are annotated according to a wellspecified coding scheme (e.g., De Wever, Schellens, Valcke, \& Van Keer, 2006; Rourke, Anderson, Garrison, \& Archer, 2000), and then statistical methods are used to characterize aggregate behaviours or compare them across conditions. Although they provide mathematically grounded methods for sampling, computing reliability and making comparisons, statistical analysis risks obscuring the sequential structure and situated methods of the interaction. Coding assigns meaning to an act as an isolated unit, and therefore either does not take the situative context of this act into account or fails to record the contextual evidence on which the analyst relied in making a judgment. Aggregation of codes into frequency counts obscures the sequential methods by which media affordances are used in particular learning accomplishments, making it more difficult to identify important design elements at the same temporal and spatial grain as the actual interaction itself. In contrast, analyses of interaction document the actual practices of learning by attending to the sequential structure of the interaction, producing detailed descriptions that are deeply situated in the medium of interaction. 
However, sequential analyses are often time consuming to produce and difficult to generalize to different media or groups. A microanalysis can capture sequential properties because analysis is focused on short interactions that an analyst can view and review, but progressively larger structures escape its grasp. The family of methods loosely classified as "exploratory sequential data analysis" (Sanderson \& Fisher, 1994) address some of these concerns with computational support for statistical and grammatical means of scaling up sequential analysis (Olson, Herbsleb, \& Rueter, 1994; Reimann, 2007). In this spirit, we also seek to retain relevant features of interaction but make interaction visible over larger scales and across temporal and spatial (media) dimensions.

\section{Analytic Representations}

The different environments and media under examination have spawned multiple environment- and medium-specific analytic representations. For example, ethnography relies to a large extent on freeform notes taken by observers. Studies of conversation have used simple transcripts of utterances (Roschelle, 1992) and more detailed transcripts using Jeffersonian notation (Sacks et al., 1974). Video has become the standard recording medium for studies of practice (Jordan \& Henderson, 1995; Koschmann et al., 2004). Video analysis tools (e.g., Pea, 2006; Woods, 2006) provide support for exploring and annotating video records, but the annotations are tied to the video medium. Online interaction simplifies the creation of transcriptions: software tools can record a detailed and comprehensive log of an interaction in a manner amenable to analysis (Bruckman, 2006; De Wever et al., 2006; Martinez, Dimitriadis, Rubia, Gomez, \& de la Fuente, 2003). However, online media introduce different asynchronic settings and hide the production of contributions (Clark \& Brennan, 1991), leading to different demands on analytic representations. Analysis of the simultaneous use of many communication media and channels has relied on ad hoc, eclectic representations (see, for example, Hmelo-Silver, 2003; Suthers, 2006a). Because interaction relies on many different semiotic resources, analysis of interactional processes must be sensitive to the social affordances of the specific medium being analysed, yet also be applicable across multiple media in order to facilitate dialog between researchers. This introduces a pair of related challenges to the creation of representations for analysing online interaction: they must be media indifferent but simultaneously media aware. A workable representation needs to be independent of the form of the data under analysis, but also maintain a record of how people make use of the specific affordances of media, supporting an analytic dialogue between theoretically motivated representations and source data (Duranti, 2006). This is required to allow analysis to speak to design and empirically drive the creation of new, more effective media.

Based on considerations discussed above, we developed an analytic approach that draws on other interaction analysis methods, but it uses generalized concepts of interaction elements and structures that are independent of any particular 
medium. The next two sections describe the theoretical foundations for our analytic representation, and how it is constructed and used.

\section{FOUNDATIONAL CONCEPTS}

\section{Uptake}

Collaborative learning is a joint process of meaning-making. In order to analyse this process we need to document the interactionally significant acts that mark out the participants' development of intersubjective understandings. Based on the requirements described above, we need a unit of interaction that abstracts from media-specific concepts such as temporal adjacency or message replies, is applicable to the wide variety of temporal, spatial and notational properties of media, and is usable for tracing the entwinement of individual and intersubjective trajectories of meaning-making.

At a fundamental level, collaborative meaning-making is only possible when contributions of each participant are encountered and transformed by other participants. Accordingly, our fundamental unit of analysis is uptake, the act of a participant taking traces of prior or ongoing action (e.g., expressions of information, attitudes and attentional orientation; whether ephemeral as in speech or persistent as in writing) forward into an ongoing process of meaning-making (Suthers, 2006a). This unit of analysis is media independent, and can reflect actions at multiple temporal or spatial scales. Uptake is interpretative: some particular aspect of the object is brought forth and given (further) meaning as a transformed object. Uptake is a transitive act, in that it always is oriented towards the taken-up as its object, which is foregrounded by the act as being relevant and becomes available as the object of future uptake. Finally, a participant can take up one's own prior traces as well as those of others. Therefore uptake as a fundamental unit of analysis is applicable to both intrasubjective and intersubjective meaningmaking. An individual working through ideas via mental processes and external notations has access to his or her uptake across as well as within these media, but in the social realm only visible acts can foreground and interpret prior traces.

We can see evidence of uptake in the coherence of conversation-each contribution is interpretable as selecting some aspect of the foregoing conversation and bridging from that to potential continuations of the conversation. Even more explicitly, a reply in a threaded discussion demonstrates the author's selection of a particular message as having relevance for further participation. Uptake is related to other units of interaction analysis, but is a more general conception. The "thematic connections" of Resnick, Salmon, Zeitz, Wathen, \& Holowchak (1993) are an example of uptake, although our conception allows for non-linguistic forms of expression, and for other kinds of interpretative acts in addition to argumentative ones. Uptake is related to but is broader than the concept of transactivity, which is often defined as reasoning that operates on the reasoning of one's partner or peers or even of oneself (Azmitia \& Montgomery, 1993; Teasley \& Roschelle, 1993). The transactivity literature focuses on interactional contexts in which a contribution is explicitly directed towards an identified other, as in (for example) 
Berkowitz \& Gibbs' (1979) coding categories for dyadic discussion. Uptake is broader because it includes situations where an actor takes up a trace of prior participation of another actor without the necessity of either person knowing that the other exists (as happens in distributed asynchronous networks of actors such as "online communities"). Also, while transactivity is sometimes treated as a property of individual utterances that can be identified by observing the other-directedness of the utterance (Azmitia \& Montgomery, 1993; Berkowitz \& Gibbs, 1979), one cannot assert uptake as a property of an individual act: it is first identified in terms of contingencies between acts. However, uptake and transactivity are compatible concepts.

Uptake is an abstraction that we as analysts use to identify interactionally significant relationships between acts. Participants don't engage in the abstract act of uptake; they perform concrete actions that they affirm (through subsequent acts) as the accomplishment of recognizable activity (Garfinkel, 1967). Similarly, the analyst's identification of uptake is a bridge from the rich web of contingencies between acts to the recognition of activity such as argumentation, knowledge building, etc. Therefore, uptake analysis begins with the identification of these contingencies between concrete actions.

\section{Contingency Graphs}

Our primary representational tool for identifying uptake is the contingency graph. A contingency graph is an abstract transcript of an interaction that documents participant actions and the identifiable contingencies between them. These contingencies provide evidence for uptake. The elements of a contingency graph are motivated by two theoretical assumptions about the nature of mediatedmediated collaborative interaction.

- Coordination: Efforts to coordinate between the personal and social realms are enacted through media, including expressions and perceptions (Hutchins, 1995).

- Nested sequential structure: The sequential structure of these coordinations at successively coarser granularities is significant to understanding how participants order and make sense of their world (Garfinkel, 1967).

The first assumption, coordination, allows us to select analytically interesting actions. Any empirical analysis must be built upon observable events. We assume that an analyst is interested in deliberate acts, not just any physical event. Therefore, the analyst will examine the ongoing stream of events and identify those that appear to be coordinations between the personal and public realms. A coordination may be any expression in verbal, gestural, or written media-we assume that the participant is attempting to coordinate the external media representation with the private (mental) representation in order to communicate. We also assume that the perception of a representation (e.g., hearing a spoken contribution, seeing a gesture, or reading a message) has some effect on the perceiver's private representation and so also constitutes a coordination. In creating 


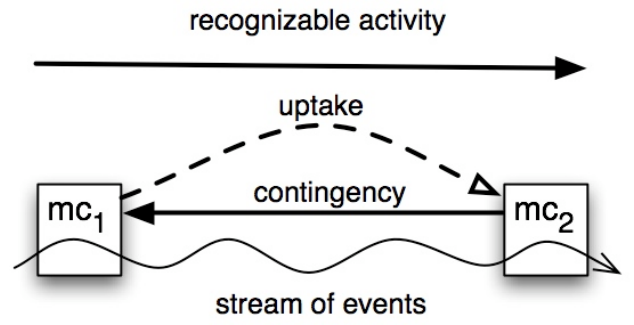

Figure 3. Schema for analytic inferences from contingencies between coordinations through uptake to recognizable activity

a contingency graph, an analyst identifies potentially interesting media coordinations.

The second theoretical assumption leads us to look for evidence of sequential structure in the media coordinations. If a media coordination $\mathrm{mc}_{2}$ is to be understood as taking up the contribution of a prior coordination $\mathrm{mc}_{1}$, then there must be some observable relationship between the media coordinations. For example, mc2 might be the act of reading a discussion message posted in mc1. Therefore, we further ground the uptake analysis in empirical evidence by identifying contingencies between media coordinations that might be relevant to the identification of uptake. Contingencies can be found in media-level, representational, and semantic relationships between media coordinations: these will be discussed below. In our example, mc2 (reading the message) is contingent on the message having been posted ( $\mathrm{mcl}$ ).

The contingency graph representation takes the form of a directed acyclic graph consisting of media coordinations and the contingencies between them (see Suthers, 2006a for a formal definition) on which we may layer analytic interpretations. Figure 3 shows the schema of basic analytical elements of the contingency graph representation. Contingencies provide evidence that uptake may exist, but not all contingencies as defined at the media level need be uptake. Contingencies reflect the myriad of ways in which human action is embedded in and sensitive to the environment and history of interaction. We identify as uptake those relationships that evidence participants' orientation towards traces of prior acts as having relevance for ongoing participation (recognizable activity).

The contingency graph provides resources for analysis by offering potential instances of uptake and grounding analysis in empirical media coordinations. The representation can support multiple methods of analysis, is amenable to computational support and visualization, and is meant as a boundary object for discussion and collaboration across different analytical traditions. Specifying contingencies makes it easier to distinguish the evidence for uptake from its interpretation. Contingencies provide evidence that one or more media coordinations played a role in enabling another media coordination, independently of our interpretation of the interactional significance of this relationship. This helps 
multiple analysts collaboratively review their observations and interpretations and facilitates trans-disciplinary discussions.

\section{CONSTRUCTING A CONTINGENCY GRAPH}

This section describes the practical tasks involved in producing a contingency graph and discussion of related issues. Then, interpretation of the graphs will be illustrated by examples from our analysis work in the next section. Although the tasks are described in sequence, in practice the process may iterate between these tasks, and may either be driven by specific analytic goals or may be more exploratory in nature.

\section{Identifying Media Coordinations}

A contingency graph is built on observed media coordinations. Media coordinations are a more general form of elements from other analytical methods, such as verbal or written contributions or workspace manipulations. Media coordinations are represented as vertices in the contingency graph. We call these vertices fixed points since they constitute points of departure for analysis that are well grounded in the data. Fixed points are anchored in media coordinations that can vary in granularity from a single instant to a period of time. The fixed point's anchor should be specific enough to allow the analyst to return to the media action as accounted in the data record. As in other interaction analysis methods, the source data is always the final authority.

Some media coordinations are easy to identify. When analysing spoken conversation or computer-mediated communication, utterances and messages, respectively, are obvious candidates for media coordinations. The creation of an object in a shared workspace is similarly easy to identify. We use the general term expressions to refer to media coordinations of this nature. Other media coordinations are less explicit. For example, if two items are placed near each other in a workspace this may be an expression of relatedness (Dwyer \& Suthers, 2006; Shipman \& McCall, 1994), but may also be arbitrary and unrelated to the content of the interaction.

Perceptions (e.g., hearing or reading another's expression) are another form of coordination between the personal and public realms. With asynchronous data, one cannot assume that each participant perceives every contribution and does so at the time that it is produced or displayed. Therefore our abstract transcript representation allows for explicit specification of evidence for perceptions as another form of media coordination. It is difficult to identify the exact content of a perception, but it is sufficient to mark the perception event as coordination of interest. Researchers interested only in public behaviour can use perceptual media coordination to narrow the temporal scope of uptake of the perceived contribution. Researchers interested in cognitive claims about individual learning may attempt to infer mental state based on other evidence, including contingencies. In either case, 
making the observed evidence for perceptual coordinations explicit supports the research.

\section{Identifying Contingencies}

The complementary task to identifying media coordinations is to identify and document the contingencies between media coordinations. A contingency represents a grounded assertion that the media coordination identified by one fixed point enabled or played a role in the media coordination identified by another fixed point. Contingencies map out the sequential unfolding of the interaction as it is embedded in its environment.

Two or more media coordinations can participate in a contingency relationship. Contingencies are directional and point backwards in time. A contingency expresses how one coordination is contingent on one or more prior media coordinations. If multiple coordinations are contingent on a single coordination, then multiple contingencies are specified. If $\mathrm{mc}_{2}$ is contingent on $\mathrm{mc}_{1}$ then we are claiming that $\mathrm{mc}_{1}$ enabled $\mathrm{mc}_{2}$, but there is no assertion that $\mathrm{mc}_{1}$ caused $\mathrm{mc}_{2}$. Contingencies underlie relationship types from other sequential data analysis methods, such as "adjacency pairs," "reply," "thematic connections," etc., and are candidate evidence for uptake. We have used three types of contingences as evidence for uptake in our work. Starting with the most concrete they are media dependencies, representational association, and semantic relatedness. These are discussed below along with examples.

\section{Media Dependency}

The most concrete contingencies are media dependencies - one action in the media could not have taken place in the absence of a previous action. A reply in a threaded discussion depends on the prior existence of the message being replied to, and modifying an element of a shared workspace depends on the previous act of creating the element. However, care must be taken not to fall into the trap of conflating the representational vocabulary with the steps in the interaction. Consider a reply in a threaded discussion. The reply message is dependent on the message being replied to, but in terms of contingencies between coordinations it is more accurate to say that the creation of the reply message is contingent on the author's perception of the message being replied to. For example, an analysis to be given shortly will contrast the reply structure of thread (Figure 4) with the dependency structure (including perceptions) from which we inferred uptake (Figure 5). Nodes with letters such as $1 \mathrm{a}, 2 \mathrm{~b}$, etc. represent media coordinations evidenced by message read events. When these perception-related media coordinations are included, we see that participant 3's posting (fixed point 7) is not only related to the single message being replied to, but is the result of a series of reads that encompasses two subthreads of the discussion (and in fact, of another discussion not shown). 


\section{Representational Association}

The second type of contingency is representational association. The use of similar representational attributes is often used to indicate relatedness (Dwyer \& Suthers, 2006; Medina \& Suthers, 2008). The representations can have similar visual attributes (e.g., colour or type face) or they can be grouped together or aligned spatially. Temporal proximity can also indicate relatedness-expressions that follow each other closely are often part of the same exchange. Each of these indications of relatedness can imply a contingency. Referring to examples to be given shortly, temporal proximity is part of our evidence for the contingency of 7 on $2 b, 4 b, 3 b, 5 b$, and $6 a$ in Figure 5: the message reads occurred immediately before the posting of a new message. In Figure 6, spatial connectivity is our evidence for the contingency of perception 20a on 19: a participant opened an object (20a) that has a link to it (created in 19), so we assume that the link was also perceived.

Representational association can also consist of repeated words and phrases indicating a contingency on the media coordination in which they were introduced. This can sometimes be easy to identify, for example when copy and paste is observed, or a phrase is typed soon after reading it. However, in general it may be more difficult to identify the original source of any content or to determine whether or not its re-use is actually dependent on the prior use.

\section{Semantic Relatedness}

The final type of contingency is semantic relatedness: the content of a coordination may have a semantic relationship to the content of another coordination. See for example the contingency of 7 on 20a in Figure 6: the text posted in 7 refers to a disease hypothesis last taken up in 20a. Semantic contingency can be difficult to identify and is often open to debate. In general, representational and semantic dependencies are more convincing if convergent evidence exists (e.g., temporal proximity and semantic relatedness co-occur, as in the example just given).

\section{Documenting other media elements}

A contingency graph is a partial transcription of an interaction. It may be necessary to record additional information to conceptualise the interaction. This additional information can annotate or augment the contingency graph formalism. In our first example, the reply structure of a threaded discussion is an important resource for understanding the participants' view of the medium, and so is included in Figures 4 and 5. In the study of our second forthcoming example, interaction was asynchronous (Suthers, Vatrapu, Medina, Joseph, \& Dwyer, 2007; Suthers et al., 2008). In order to identify which representational elements each participant had available at any point in time, we indicated when workspace updates displayed new data from their partner, visualized as vertical bars in Figure 6. 


\section{Iteration}

Production of the contingency graph can be an iterative process: multiple passes through the data identify additional elements and provide new insights into the interaction. The formalism of the contingency graph provides support for this process. New fixed points and contingencies can be continually added to the graph, and the graph can grow arbitrarily complex to reflect a deepening knowledge of the data. Additionally, repeated iterations may identify new types of representational elements, media coordinations, and contingencies.

\section{Robustness}

The quality of the analysis is proportional to the richness of the data: the method is robust in that one can work with the data available. In our work with threaded discussions for online courses we only have log entries for when a message was created and when a user opened a message. Other media coordinations such as scrolling are not logged. On the other hand, the software configuration of our second example provides a complete record of every action on the shared representation, and we also have a video capture of the computer screen from each client. The richness of the latter data has allowed us to examine interaction at a much finer grain. Nonetheless, the threaded discussion data is sufficient for coarser grained analysis. The possibility that further data can always be considered has the implication that the graph can never be considered "complete," except with regard to particular representational elements (e.g., it is possible to claim that every discussion posting has been recorded as a fixed point). Therefore, one must be cautious about asserting that a practice or pattern never occurs.

\section{EXAMPLES OF DISCOVERY BY UPTAKE ANALYSIS}

In the following sections we present examples of using the uptake analysis framework to expose interactions in two dissimilar online environments. The data sources for both analyses included software logs stored in database tables. The use of $\log$ data provided a reliable format for determining the actor, exact timing, and form of media coordinations and for automated identification of certain classes of contingencies between them. In addition, video screen capture recordings were used to determine contingencies in the second example below.

\section{Exposing students' learning activities in online discussions}

Our first example demonstrates the uptake analysis methodology applied to threaded discussion postings from a graduate-level course. After reading a paper on socio-constructivist, socio-cultural, and shared cognition theories of collaborative learning (Dillenbourg, Baker, Blayne, \& O'Malley, 1996), a student facilitator suggested that students write "grant proposals" to evaluate learning in the course itself, and discuss how their choice of theory would affect how they approach the 


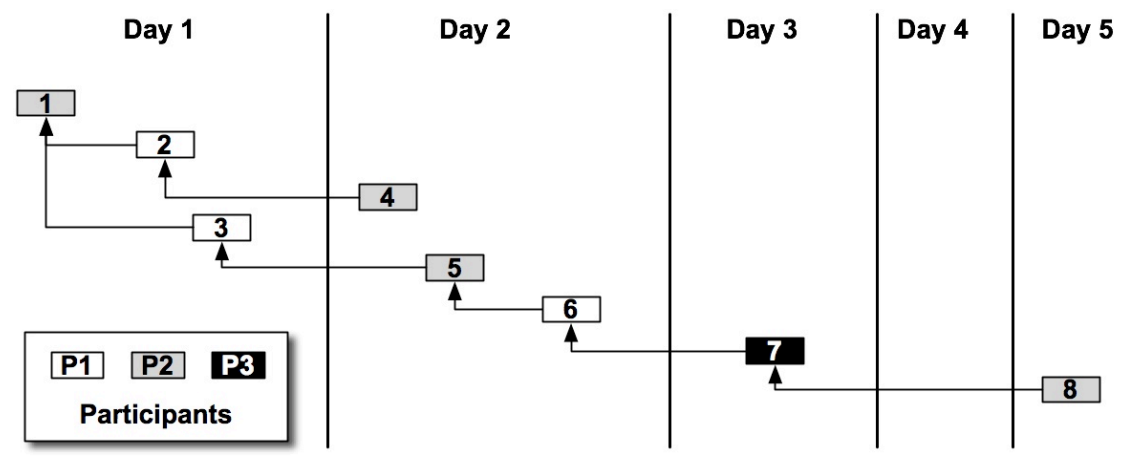

Figure 4. Discussion reply structure organized temporally

evaluation. Working groups addressed this idea in separate discussion forums. We examine one thread of one group's discussion (shown in Figure 4 and Table 1). The process by which we built a contingency graph for this interaction is illustrative of the graph creation process, and the resulting contingency graph makes several interesting aspects of student activity available for further analysis.

Table 1. Excerpts from messages

\begin{tabular}{|c|c|c|c|}
\hline 1 & $\mathrm{P} 2$ & $\begin{array}{l}9 / 23 \\
3: 39\end{array}$ & $\begin{array}{l}\text { "... In your first post, your needs assessment seems to be talking about } \\
\text { socio-cognitive rather than socio-cultural..." }\end{array}$ \\
\hline 2 & $\mathrm{P} 1$ & $\begin{array}{l}9 / 23 \\
11: 15\end{array}$ & $\begin{array}{l}\text { "What is the "socio-cognitive" approach? I'd like to read more about this } \\
\text { approach since I am not familiar with it. I was really interested in the } \\
\text { socio-cultural approach because it seems to imply that intellectual } \\
\text { development is directly related to socialization." }\end{array}$ \\
\hline 3 & P1 & $\begin{array}{l}9 / 23 \\
11: 31 \\
\end{array}$ & $\begin{array}{l}\text { "I didn't see any description of the "socio-cognitive" approach in the } \\
\text { assigned readings. I was not familiar with this approach..." }\end{array}$ \\
\hline 4 & P2 & $\begin{array}{l}9 / 24 \\
2: 33\end{array}$ & $\begin{array}{l}\text { "...what is unique about socio-cultural (or CHAT - cultural historical } \\
\text { activity theory) is the emphasis on cultural and social context. But you } \\
\text { are right, it does give an account of individual cognitive change as a } \\
\text { function of social interaction..." }\end{array}$ \\
\hline 5 & $\mathrm{P} 2$ & $\begin{array}{l}9 / 24 \\
2: 34\end{array}$ & $\begin{array}{l}\text { "...Sorry, I meant socio-constructivist (though I have used socio- } \\
\text { cognitive to include the former)...." }\end{array}$ \\
\hline 6 & P1 & $\begin{array}{l}9 / 24 \\
3: 35\end{array}$ & "Thank you - that clears it up for me! :)" \\
\hline 7 & P3 & $\begin{array}{l}9 / 25 \\
10: 14\end{array}$ & $\begin{array}{l}\text { "I noticed that several of our grant proposals mixed up socio-cognitive } \\
\text { for the socio-constructivist. I was thrown a little at first. Anyone know } \\
\text { where the confusion stems from?" }\end{array}$ \\
\hline 8 & P1 & $\begin{array}{l}9 / 27 \\
2: 35\end{array}$ & $\begin{array}{l}\text { "...any constructivist approach is cognitivist. Constructivism includes } \\
\text { among its explanatory concepts the ideas that people build internal } \\
\text { (cognitive) structures...." }\end{array}$ \\
\hline
\end{tabular}

The first and obvious set of media coordinations we identified were the acts of posting messages. The reply structure between them documents a set media 
dependencies - a reply message is contingent on the existence of the message being replied to. In order to understand the temporal structure of the interaction, we organized these media coordinations alone a timeline based on the time of each message posting (Figure 4). The episode took place over 5 days, indicated in Figure 4 by vertical lines for midnight of each day. Messages 2 and 3 appear in the threading structure as two separate replies to 1 , but examination of the message content (Table 1) shows that these two messages seem to be two parts of a single question on the part of the student (P1).

We then considered the events in which participants read messages. Figure 5 shows the contingency graph produced by adding read events along with the associated contingencies. In this case we assert that a read event is contingent on the pre-existence of the item being read (a media dependency), and we assert that posts that closely follow reads are likely contingent on the content of the read message (temporal contingency). Content analysis (not shown here) bears out this second assertion. In this graph, read events are indicated by X1...Xn, Y1...Yn, and labels ending with a letter (1a, etc.). Dashed lines indicate intrasubjective contingencies (a person's post actions are dependent on their own read actions). Days 4 and 5 are omitted for space reasons.

The graph in Figure 5 exposes the mechanism underlying the divergence and eventual re-convergence of the discussion. In message 2, P1 has expressed interest in a concept ("I'd like to read more about this approach ..."), and then went off to read other messages (read events X1 ... Xn). Message 3 starts with "I didn't see any description of the socio-cognitive approach ...", the concept of interest in message 2. P1 is forced to post two separate replies ( 2 and 3 ) because the medium does not allow her to edit her first reply, and this opens up the possibility of a divergent discussion. By following a strategy of reading and replying to each message one at a time ( $\mathrm{read} /$ write pairs $2 \mathrm{a} / 4$, and $3 \mathrm{a} / 5), \mathrm{P} 2$ continues the split that

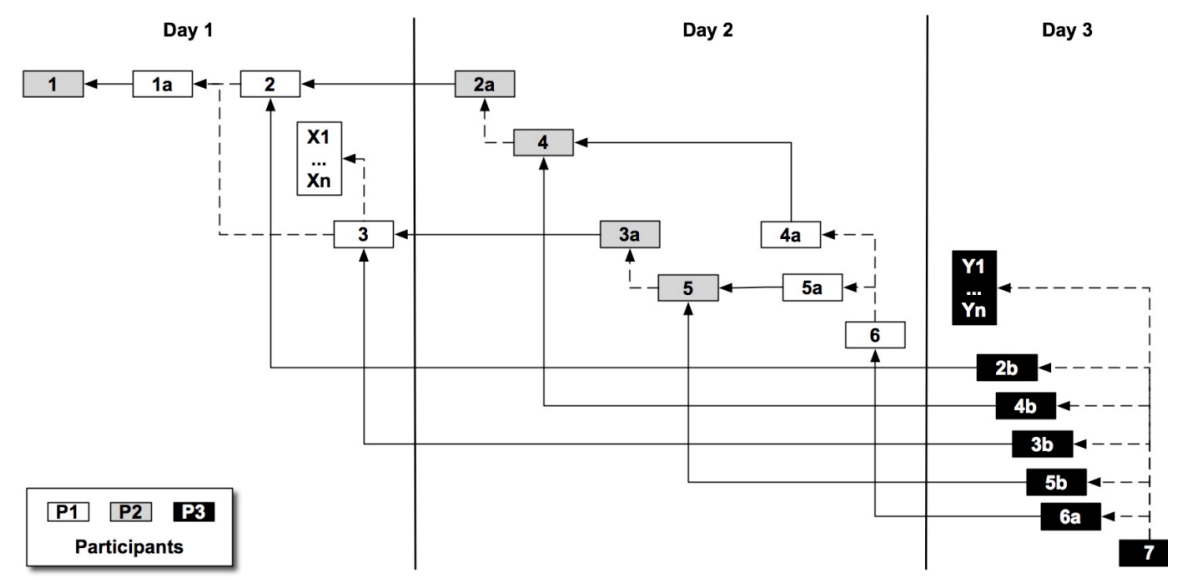

Figure 5. Discussion structure with selected read actions added. 
P1 started with messages 2 and 3. In contrast, by following a strategy of reading all messages before replying, P1 re-integrates the split threads $(4 a / 5 a / 6)$ with a message thanking P2. Similarly, P3 replies after reading all the existing messages in the thread. However, the reply structure of the discussion tool does not allow either of these convergences to be expressed in the medium: P3 must choose one message to reply to, so she replies to the last one she read. Her message seems odd as a reply to the "thank you," as it refers to the concepts under discussion in the broader context of "several of our grant proposals." In a sequence of reads of messages in another discussion forum (Y1...Yn) about an hour before entering this forum, P3 had read through the grant proposals. Viewing the reply structure alone, P3's comment appears as an isolated intervention in a dialogue between P1 and P2, but viewing a contingency graph that includes read, P3's comment is seen as a highly integrative contribution resulting from careful perusal of the discussions.

The contingency graph captures aspects of the coherence of the mediated interaction that are not apparent in the media trace itself (e.g., the threaded reply structure in this example). Although some of this coherence can be recovered through analysis of quoting practices (Barcellini, Détienne, Burkhardt, \& Sack, 2005), our analysis goes further to include (for example) lexical and temporal evidence for coherence, evidence that can also be partially automated (e.g., Rosé et al., in press). This ability to identify trajectories that are independent of yet influenced by media structures is an important strength of the method.

\section{An Interactional Pattern}

Our next example is derived from a study of collaborative interaction with evidence maps. Participants are presented with information about a mysterious disease on the island of Guam. Information pages suggest alternative hypotheses concerning the cause, and provide evidence for and against each cause. Evidence is distributed across participants in a "hidden profile" (Stasser, 1992), requiring that they share information to come to an optimal conclusion, and enabling us to trace out their information sharing. Participants interact via a computer environment with asynchronous updating (delayed transmission of the partner's work) to simulate online learning, as illustrated in Figure 2. The original study was an experimental comparison of three software environments (see Suthers, Vatrapu, Medina, Joseph et al., 2007; Suthers et al., 2008). The analysis reported here was done to understand how two participants converged on the conclusion that aluminium is probably not the cause of a disease under consideration. Since the evidence for this cause had been given to one person and the evidence against it to the other, information sharing was required for them to converge on the same conclusion. We asked: how was this information sharing and convergence accomplished with the media resources provided by the software environment?

Construction of the contingency graph began with the essay writing events that indicated there was convergence (e13 and e18 in Figure 6), and worked backwards to identify contingencies on prior events (hence the numbering of fixed points in the figure increases going backwards in time). We organized fixed points for both 
EXPOSING INTERACTIONAL PROCESSES IN ONLINE LEARNING

manipulations and perceptions of workspace elements along a timeline and added contingencies based on media dependencies and temporal proximity. Additionally, we coded each contribution based on its content and then examined sequences of media coordinations that referenced similar information items. In this example, we focused on exchanges of information about aluminium. 


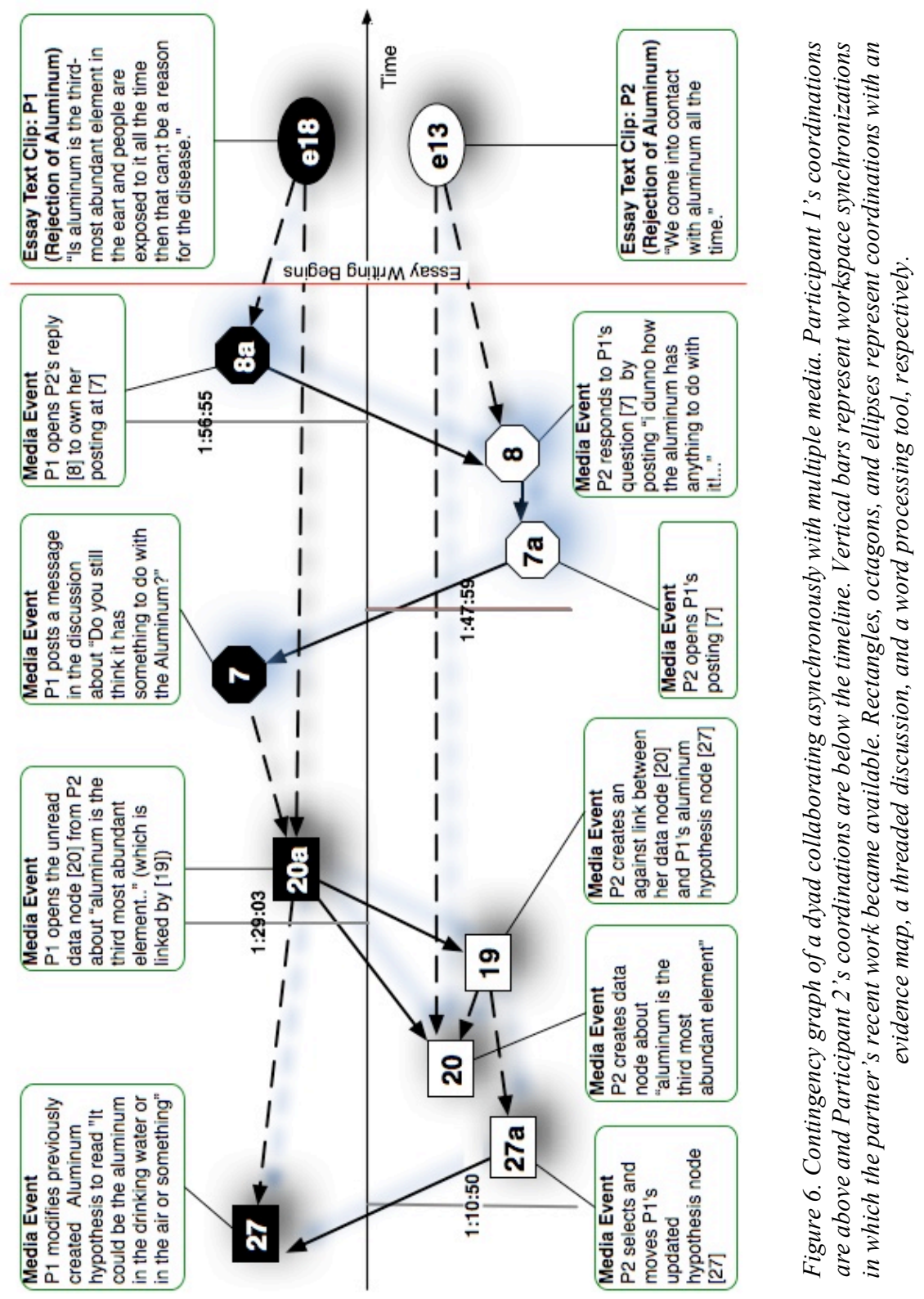


The resulting contingency graph was very complex, but it allowed us to examine the patterns that produced convergence in the participant's conclusions. We discovered an interesting interactional pattern that goes beyond simple information sharing. Figure 6 shows the portion of the contingency graph that evidenced this pattern of uptake. The information that "aluminium is the third most abundant element" and that this contradicts aluminium as a causal agent has been successfully shared via the evidence map (media coordinations 27, 27a, 20, 19 and 20a). However, participants interacted again over 20 minutes later in the session (7-7a-8-8a) to confirm their interpretation. By exposing this dual round trip structure, the uptake analysis enabled us to hypothesize an interactional patternwhich we call the "W"- in which information is first shared in one exchange, and then agreement on a joint interpretation of this information is accomplished in a second exchange. The analysis also helped us discover that participants accomplished the confirmation by moving to a different interactional medium, the threaded discussion - a result that is consistent with observations from other data that participants use the linguistic medium when second-order discussion is required (Suthers, 2006a).

\section{DISCUSSION}

The initial motivation for developing the contingency graph formalism was to support our analysis of collaborative knowledge construction through computer media. As this work progressed, we removed implicit assumptions about synchronicity and availability of contributions from the notation. We also realized that we could use the contingency graph as a boundary object between our different analysis methods. For example, we used the contingency graph to create aggregate statistics of interactions and their relationship to the media (Suthers, Vatrapu, Medina, Joseph et al., 2007; Suthers et al., 2008), and to examine the sequential structure of interaction (Suthers, Dwyer, Medina, \& Vatrapu, 2007). The graph allowed us to trace asynchronous interaction between pairs of participants back from aspects of their essays that we wanted to explain. Our most recent analysis of the data (Suthers, Vatrapu, Medina, \& Dwyer, 2007) bridged statistical and sequential approaches by algorithmically identifying instances of an interaction pattern we refer to as a "round trip" and then applying statistical tests on their frequency across experimental conditions.

We are often asked how long an analysis takes, and what tools we used. Time estimates that are predictive of future work are not yet possible, because the analyses reported in this chapter took place concurrently with extensive discussions in which we developed the theoretical and practical basis for the framework. These discussions took place over many months with multiple revisions of the analyses, although subsequent analyses have been done in several days. Visualizations of contingency graphs were constructed using general-purpose tools such as a MySQL database, Excel ${ }^{\mathrm{TM}}$, Visio ${ }^{\mathrm{TM}}$, and Omnigraffle ${ }^{\mathrm{TM}}$. Software tools tailored to this task will support more efficient analysis. A key factor in the type of analysis tool we envision is the format of the source data. To date we have used software logs containing important identifiers for users, time, media objects, and content in constructing and working with contingency graphs. Constructing graphs from other 
forms of recorded interaction such as video of face to face interaction is possible to the extent that aspects of the data can be tagged with respect to time, actors, media objects, and content.

There are multiple benefits to the contingency graph as a transcript notation. First, the notation is independent of the interaction medium and can be applied to face-to-face and online interactions as well as interactions that take place in multiple media. The use of generic media coordinations allows the inclusion of a whole range of communicative actions, including perceptions and interactionally constructed representational elements. The concept of contingency extends the concepts of utterance and adjacency pair to online and asynchronous media and accounts for cases where media coordination is the result of multiple, previous media coordinations. Second, the notation can be used to document and interpret the sequential structure of the interaction and can also be coded or searched to provide data for statistical analyses. Third, the contingency graph adapts to the density of the source data. High-fidelity data can be used to produce a dense graph that can be subject to detailed analysis. On the other hand, sparse data will produce a sparse graph but will still support limited analysis. Fourth, the graph data structure is open-ended-additional data can always be added, although this does imply that scepticism about the completeness of the graph should be maintained. Fifth, grounding in explicit media coordinations allows analysis of correlations between interaction patterns and the media affordances that shape them. Finally, the formalism of the graph structure supports building tools to manage its complexity and is amenable to algorithmic analysis and data mining techniques. Further development of automated analysis and visualization tools will help realize the value of the notation to practitioners as well as researchers in providing a window on distributed interaction: the "pulse" of the online "classroom."

A limitation of the framework is that, in focusing on observed interaction, it does not explicitly acknowledge the cultural or historical situatedness of the participants, or address identity and community, except where these constructs might be recorded in terms of prior interaction. Many theoretical and practical issues remain to be worked out. A pressing task is to extend the contingency graph formalism to better incorporate composite media coordinations and the possible ambiguity of contingencies. A complete explication of these two items is necessary to extend the potential algorithmic support provided by the contingency graph structure. The greatest practical need is to develop software tools to help construct and use the contingency graph. The need for improved analysis tools is a recurring theme (Sanderson \& Fisher, 1994), and the size and density of the potential data sets exacerbates this need. Alternative visual representations should be explored. Although we display the contingency graphs as visual node-link graphs, one should not conflate the abstract representation with its potential visualizations. For example, contingency graphs can be used to generate both node-link displays of uptake and CORDTRA-style representations of episodes of activity (chronological representations of discourse and tool-related activity; Hmelo-Silver, 2003; see also Hmelo-Silver, Chernobilsky, \& Nagarajan, this volume). An important aspect of evaluating this framework will be to determine how well it scales to the types of 
interactions and media that are of most interest, including larger groups across longer time scales. Manual identification of media coordinations and contingencies is time-consuming at present, but with improved automation it might be possible to generate contingency graphs for larger online communities over the course of months or even years. Finally, the value of this framework in supporting multiple analytic traditions and producing "boundary objects" for research can only be realized in collaboration with other laboratories undertaking analysis of collaborative interaction.

\section{ACKNOWLEDGMENTS}

This work was supported by the National Science Foundation under award 0093505. Any opinions, findings, and conclusions or recommendations expressed in this paper are those of the authors and do not necessarily reflect the views of the National Science Foundation.

\section{REFERENCES}

Allen, I. E., \& Seaman, J. (2005). Growing by Degrees: Online Education in the United States, 2005. Needham, MA: Alfred P. Sloan Foundation.

Aviv, R., Erlich, Z., Ravid, G., \& Geva, A. (2003). Network Analysis of Knowledge Construction in Asynchronous Learning Networks. Journal of Asynchronous Learning Networks, 7(3), http://www.sloan-c.org/publications/jaln/v7n3/v7n3_aviv.asp.

Azmitia, M., \& Montgomery, R. (1993). Friendship, Transactive Dialogues, and the Development of Scientific Reasoning. Social Development, 2(3), 202-221.

Baker, M. (2003). Computer-mediated argumentative interactions for the co-elaboration of scientific learning tasks. In J. Andriessen, M. Baker \& D. D. Suthers (Eds.), Arguing to Learn: Confronting Cognitions in Computer-Supported Collaborative Learning Environments. (pp. 47-78.). Dordrecht: Kluwer.

Barcellini, F., Détienne, F., Burkhardt, J.-M., \& Sack, W. (2005, November 06 - 09, 2005). Thematic coherence and quotation practices in OSS design-oriented online discussions. Paper presented at the Proceedings of the 2005 International ACM SIGGROUP Conference on Supporting Group Work (GROUP '05), Sanibel Island, Florida, USA.

Beck, I. L. (1997). Questioning the Author: An Approach for Enhancing Student Engagement with Text. Delaware: International Reading Association.

Berkowitz, M. W., \& Gibbs, J. C. (1979). A Preliminary Manual for Coding Transactive Features of Dyadic Discussion.

Bronckart, J. P. (1995). Theories of action, speech, natural language, and discourse. In J. V. Wertsch, P. D. Rio \& A. Alvarez (Eds.), Sociocultural Studies of Mind (pp. 75-91). New York: Cambridge University Press.

Bruckman, A. S. (2006). Analysis of Log File Data to Understand Behavior and Learning in an Online Community. In J. Weiss, J. Nolan, J. Hunsinger \& P. Trifonas (Eds.), International Handbook of Virtual Learning Environments (pp. 1449-1465). New York: Springer.

Campos, M. (2004). A Constructivist Method for the Analysis of Networked Cognitive Communication and the Assessment of Collaborative Learning and Knowledge-Building. Journal of Asynchronous Learning Networks, 8(2), c.org/publications/jaln/v8n2/v8n2_campos.asp.

Chi, M., Bassok, M., Lewis, M., Reimann, P., \& Glaser, R. (1989). Self-Explanations: How Students Study and Use Examples in Learning to Solve Problems. Cognitive Science, 13, 145-182. 


\section{EXPOSING INTERACTIONAL PROCESSES IN ONLINE LEARNING}

Clark, H. H., \& Brennan, S. E. (1991). Grounding in communication. In L. B. Resnick, J. M. Levine \& S. D. Teasley (Eds.), Perspectives on Socially Shared Cognition (pp. 127-149): American Psychological Association.

De Wever, B., Schellens, T., Valcke, M., \& Van Keer, H. (2006). Content analysis schemes to analyze transcripts of online asynchronous discussion groups: A review. Computers \& Education, 46(1), 628.

Dervin, B. (2003). Chaos, Order, and Sense-Making: A Proposed Theory for Information Design. In B. Dervin \& L. Foreman-Wenet (Eds.), Sense-Making Methodology Reader: Selected Writings of Brenda Dervin (pp. 325-340). Cresskill, New Jersey: Hampton Press, Inc.

Dillenbourg, P. (2005). Designing biases that augment socio-cognitive interactions. In R. Bromme, F. W. Hesse \& H. Spada (Eds.), Barriers and Biases in Computer-Mediated Knowledge Communication - and How They May Be Overcome (pp. 243-264). New York, NY: Springer.

Dillenbourg, P., Baker, M., Blayne, A., \& O'Malley, C. (1996). The evolution of research on collaborative learning. In E. Spada \& P. Reimann (Eds.), Learning in Humans and Machine: Towards an interdisciplinary learning science (pp. 189-211). Oxford: Elsevier.

Doise, W., \& Mugny, G. (1984). The Social Development of the Intellect, International Series in Experimental Scoial Pscychology (Vol. 10): Pergamon Press.

Duranti, A. (2006). Transcripts, Like Shadows on a Wall. Mind, Culture \& Activity, 13(4), 301-310.

Dwyer, N., \& Suthers, D. D. (2006). Consistent Practices in Artifact-Mediated Collaboration. International Journal of Computer-Supported Collaborative Learning, 1(4), 481-511.

Engestrom, Y. (2001). Expansive Learning at Work: toward an activity theoretical reconceptualization. Journal of Education and Work, 14(1).

Garfinkel, H. (1967). Studies in Ethnomethodology. Englewood Cliffs, New Jersey: Prentice-Hall.

Goodwin, C., \& Heritage, J. (1990). Conversation Analysis. Annual Review of Anthropology, 19, 283307.

Hmelo-Silver, C. E. (2003). Analyzing collaborative knowledge construction: Multiple methods for integrated understanding. Computers \& Education, 41, 397-420.

Hutchins, E. (1995). Cognition in the Wild. Cambridge, Massachusets: The MIT Press.

Jordan, B., \& Henderson, A. (1995). Interaction analysis: Foundations and practice. Journal of the Learning Sciences, 4(1), 39-103.

Koschmann, T., \& LeBaron, C. (2003). Reconsidering common ground: Examining Clark's contribution theory in the OR. Paper presented at the ECSCW 2003: Eighth European Conference on ComputerSupported Collaborative Work, Amsterdam.

Koschmann, T., Zemel, A., Conlee-Stevens, M., Young, N., Robbs, J., \& Barnhart, A. (2005). How do people learn: Member's methods and communicative mediation. In R. Bromme, F. W. Hesse \& H. Spada (Eds.), Barriers and Biases in Computer-Mediated Knowledge Communication (and how they may be overcome) (pp. 265-294). Amsterdam: Kluwer Academic Press.

Koschmann, T., Zemel, A., \& Stahl, G. (2004). The video analyst's manifesto (or the implications of garfinkel's policies for studying practice within design-based research). In Y. Kafai, N. Sandoval, N. Enyedy, A. Nixon \& F. Herrera (Eds.), Proceedings of the Sixth International Conference of the Learning Sciences (pp. 278-385). Mahwah, NJ: Lawrence Erlbaum Associates.

Martinez, A., Dimitriadis, Y., Rubia, B., Gomez, E., \& de la Fuente, P. (2003). Combining qualitative evaluation and social network analysis for the study of classroom social interactions. Computers \& Education, 41, 353-368.

Mayadas, F. (1997). Asynchronous Learning Networks: A Sloan Foundation Perspective. Journal of Asynchronous Learning Networks, 1 , http://www.aln.org/alnweb/journal/jaln_issue1.htm\#mayadas.

Medina, R., \& Suthers, D. D. (2008, June 24-28, 2008). Bringing Representational Practice From Log to Light. Paper presented at the International Conference for the Learning Sciences, Utrecht.

Moore, J. C. (2004). A Synthesis of Sloan-C Effective Practices, August 2005. Journal of Asynchronous Learning Networks, 9(3), http://www.sloanc.org/publications/jaln/v9n3/v9n3_moore_member.asp. 


\section{EXPOSING INTERACTIONAL PROCESSES IN ONLINE LEARNING}

Olson, G. M., Herbsleb, J. D., \& Rueter, H. H. (1994). Characterizing the sequential structure of interactive behaviors through statistical and grammatical techniques. Human-Computer Interaction, 9, 427-472.

Orey, M., McClendon, V. J., \& Branch, R. M. (Eds.). (2006). Educational Media and Technology Yearbook. Westport, CT: Libraries Unlimited.

Parker, P. L. (2000). Learning Technologies and their Impact on Science Education: Delivering the Promise. Australian Science Teachers Journal, 46(3), 9-20.

Pea, R. (2006). Diver. Retrieved Nov. 26, 2006, from http://diver.stanford.edu

Ramage, T. R. (2002). The 'No Significant Difference' Phenomenon: A Literature Review. Journal of Instructional Science and Technology, 5(1), http://www.usq.edu.au/electpub/ejist/docs/html2002/ramage_frame.html.

Reimann, P. (2007). Time is precious: Why process analysis is essential for CSCL (and can also help to bridge between experimental and descriptive methods). In C. Chinn, G. Erkens \& S. Puntambekar (Eds.), The Computer Supported Collaborative Learning (CSCL) Conference 2007 (pp. 598-607). New Brunswick: International Society of the Learning Sciences.

Resnick, L. B., Salmon, M., Zeitz, C. M., Wathen, S. H., \& Holowchak, M. (1993). Reasoning in conversation. Cognition and Instruction, 11(3\&4), 347-364.

Resnick, P. (2002). Beyond Bowling Together: SocioTechnical Capital. In J. M. Carroll (Ed.), HumanComputer Interaction in the New Millennium (pp. 647-672). Upper Saddle River, NJ: ACM Press.

Roschelle, J. (1992). Learning by Collaborating: Convergent Conceptual Change. The Journal of the Learning Sciences, 2(3), 235-276.

Roschelle, J. (1996). Designing for cognitive communication: Epistemic fidelity or mediating collaborating inquiry. In D. L. Day \& D. K. Kovacs (Eds.), Computers, Communication \& Mental Models (pp. 13-25). London: Taylor \& Francis.

Rosé, C., Wang, Y.-C., Cui, Y., Arguello, J., Stegmann, K., Weinberger, A., et al. (in press). Analyzing collaborative learning processes automatically: Exploiting the advances of computational linguistics in computer-supported collaborative learning. International Journal of Computer-Supported Collaborative Learning, DOI: 10.1007/s11412-007-9034-0.

Rourke, L., Anderson, T., Garrison, D. R., \& Archer, W. (2000). Methodological Issues in the Content Analysis of Computer Conference Transcripts. International Journal of the Learning Sciences, 11 .

Sacks, H., Schegloff, E. A., \& Jefferson, G. (1974). A Simplest Systematics for the Organization of Turn-Taking for Conversation. Language, 50(4), 696-735.

Sanderson, P., \& Fisher, C. (1994). Exploratory Sequential Data Analysis: Foundations. HumanComputer Interaction, 9, 251-317.

Scardamalia, M., \& Bereiter, C. (1991). Higher Levels of Agency for Children in Knowledge Building: A Challenge for the Design of New Knowledge Media. The Journal of the Learning Sciences, 1(1), 37-68.

Shipman, F. M., III, \& McCall, R. (1994). Supporting knowledge-base evolution with incremental formalization, CHI94 (pp. 285-291). Boston, MA: ACM Press.

Stahl, G. (2006a). Group cognition: computer support for collaborative knowledge building. Cambridge, MA: MIT Press.

Stahl, G. (2006b). Sustaining Group Cognition in a Math Chat Environment. Research and Practice in Technology Enhanced Learning 1(2).

Stasser, G. (1992). Information salience and the discovery of hidden profiles by decision-making groups: A "thought experiment". Organizational Behavior and Human Decision Processes, 52(1), 156-181.

Suthers, D. D. (2006a). A qualitative analysis of collaborative knowledge construction through shared representations. Research and Practice in Technology Enhanced Learning (RPTEL), 1(2), 1-28.

Suthers, D. D. (2006b). Technology affordances for intersubjective meaning-making: A research agenda for CSCL. International Journal of Computers Supported Collaborative Learning, 1(3), 315-337. 


\title{
EXPOSING INTERACTIONAL PROCESSES IN ONLINE LEARNING
}

Suthers, D. D. (in press). Empirical studies of the value of conceptually explicit notations in collaborative learning. In A. Okada, S. Buckingham Shum \& T. Sherborne (Eds.), Knowledge Cartography (pp. (24 pages)). Cambridge, MA: MIT Press.

Suthers, D. D., Connelly, J., Lesgold, A. M., Paolucci, M., Toth, E. E., Toth, J., et al. (2001). Representational and advisory guidance for students learning scientific inquiry. In K. D. F. a. P. J. Feltovich (Ed.), Smart Machines in Education: the Coming Revolution in Educational Technology (pp. 7-35). Cambridge, Massachusetts: AAAI Press, The MIT Press.

Suthers, D. D., Dwyer, N., Medina, R., \& Vatrapu, R. (2007). A framework for analyzing interactional processes in online learning Annual Meeting of the American Educational Research Association (AERA). Chicago.

Suthers, D. D., \& Hundhausen, C. (2003). An experimental study of the effects of representational guidance on collaborative learning. Journal of the Learning Sciences, 12(2), 183-219.

Suthers, D. D., Vatrapu, R., Medina, R., \& Dwyer, N. (2007). Information Sharing and Interaction in Collaborative Convergence, International Conference on Computers in Education. Hiroshima.

Suthers, D. D., Vatrapu, R., Medina, R., Joseph, S., \& Dwyer, N. (2007). Conceptual representations enhance knowledge construction in asynchronous collaboration. In C. Chinn, G. Erkens \& S. Puntambekar (Eds.), Computer Supported Collaborative Learning 2007. Rutgers: ISLS.

Suthers, D. D., Vatrapu, R., Medina, R., Joseph, S., \& Dwyer, N. (2008). Beyond threaded discussion: Representational guidance in asynchronous collaborative learning environments. Computers \& Education, 50(4), 1103-1127.

Teasley, S. D., \& Roschelle, J. (1993). Constructing a joint problem space: The computer as a tool for sharing knowledge. In S. P. Lajoie \& S. J. Derry (Eds.), Computers as Cognitive Tools (pp. 229258). Hillsdale, NJ: Lawrence Erlbaum Associates.

Toth, E. E., Suthers, D. D., \& Lesgold, A. M. (2002). "Mapping to know": The effects of representational guidance and reflective assessment on scientific inquiry. Science Education, 86(2), 264-286.

Vygotsky, L. S. (1978). Mind in society. Cambridge, MA: Harvard University Press.

Wenger, E. (1998). Communities of Practice: Learning, Meaning and Identity. Cambridge: Cambridge University Press.

Wertsch, J. V. (1998). Mind as Action. New York: Oxford University Press.

Woods, D. (2006). Transana. Retrieved Nov. 26, 2006, from http://www.transana.org

\author{
AFFILIATIONS \\ Daniel D. Suthers \\ Richard Medina \\ Ravi Vatrapu \\ Department of Information and Computer Sciences \\ University of Hawaii
}

Nathan Dwyer

SRI 